\title{
JOURNAL.RU
}

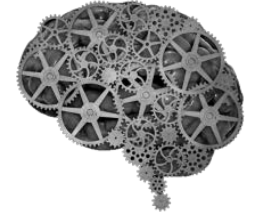

COMPANY GROUP

"INTELLEKT"

Бадриев И.Б., Макаров М.В., Мартынова О.П.

Казанский (Приволжский) федеральный университет

Казань, Россия

doi: 10.18411/lj2016-7-1-01

idsp 000001: lj2016-16-1-01

\section{Постановка геометрически нелинейной задачи об изгибе трехслойной пластины с трансверсально-мягким заполнителем}

\section{Введение}

Создание изделий авиационной и космической техники, судостроения, строительства в настоящее время неразрывно связано с применением новых конструкционных материалов и элементов конструкций из них, обладающих высокими прочностными и жесткостными характеристиками. Таким требованиям отвечают слоистые элементы конструкций, в частности, трехслойные [1-3].

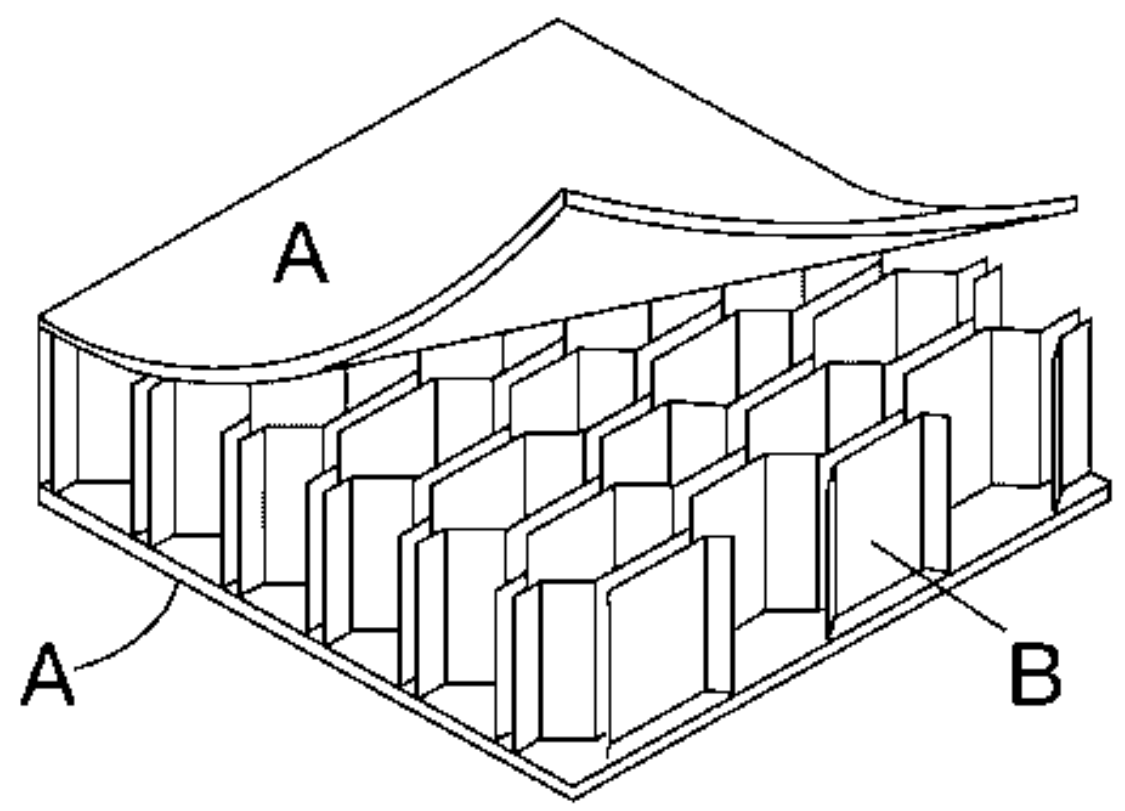

Рис 1. Общий вид трехслойной конструкции: А - несущие слои; В - заполнитель. 
Эти конструкции состоят из материалов с различными физикомеханическими свойствами - несущие слои обычно изготавливаются из материалов с высокими механическими характеристиками и предназначены для восприятия основной нагрузки; связующий слой, служащий для образования монолитной конструкции, обеспечивает перераспределение усилий между несущими слоями, выполняет функции защиты от тепловых, химических, радиационных и других нежелательных воздействий. Применение в качестве заполнителя материалов с низкими массовыми характеристиками позволяет при сравнительно небольшом увеличении веса конструкции существенно повысить изгибную жесткость. Тем самым трехслойные конструкции нашли широкое применение в качестве несущих и управляющих поверхностей летательных аппаратов, обтекателей, теплозащитных и силовых экранов, разного рода панелей и других конструктивных элементов [4-6].

Настоящая работа посвящена исследованию корректности обобщенной постановки геометрически нелинейной задачи об изгибе трехслойной пластины с трансверсально-мягким заполнителем. Обобщенная постановка для физически нелинейной и геометрически линейной задачи в виде седловой задачи, а также метод для ее решения рассмотрены в [7-11].

\section{1. Постановка задачи.}

В данной работе рассматриваются задачи об определении напряженнодеформированного состояния бесконечно длинной трехслойной пластины с трансверсально-мягким заполнителем (см. рис. 2). Ширина пластины равна $a$, толщина заполнителя составляет $2 \mathrm{~h}$, толщины несущих слоев равны $2 h_{(k)}$, где $\mathrm{k}$ - номер слоя. Исследование процессов деформирования таких элементов прежде всего диктуется необходимостью определения степени их пригодности для дальнейшего использования. 


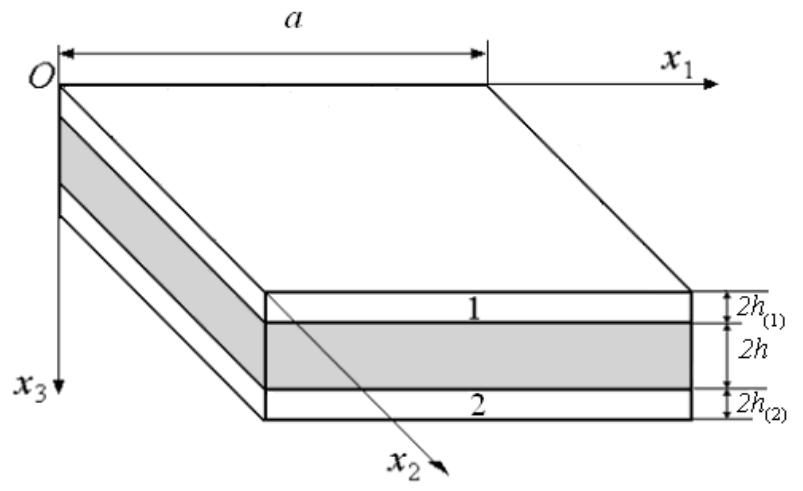

Рис 2. Бесконечно длинная трехслойная пластина с трансверсально-мягким заполнителем.

Для описания напряженно-деформированного состояния в несущих слоях используются уравнения модели Кирхгофа-Лява, в заполнителе - уравнения теории упругости, упрощенные в рамках принятой модели трансверсальномягкого слоя и проинтегрированных по толщине с удовлетворением условий сопряжения слоев по перемещениям [12-14].

В соответствии с [12-14] введем следующие обозначения (см. рис. 3): $H_{(k)}=h+h_{(k)}$ (здесь и всюду в дальнейшем предполагаем, что $\left.\mathrm{k}=1,2\right),{ }_{(k)}^{1}$, $X_{(k)}^{3}$ - компоненты поверхностной нагрузки, приведенной к срединной поверхности k-го слоя, $w^{(k)}$ и $u^{(k)}-$ прогибы и осевые перемещения точек срединной поверхности к-го слоя соответственно, $T_{(k)}^{11}, M_{(k)}^{11}-$ мембранные усилия и внутренние изгибающие моменты в k-м слое соответственно.

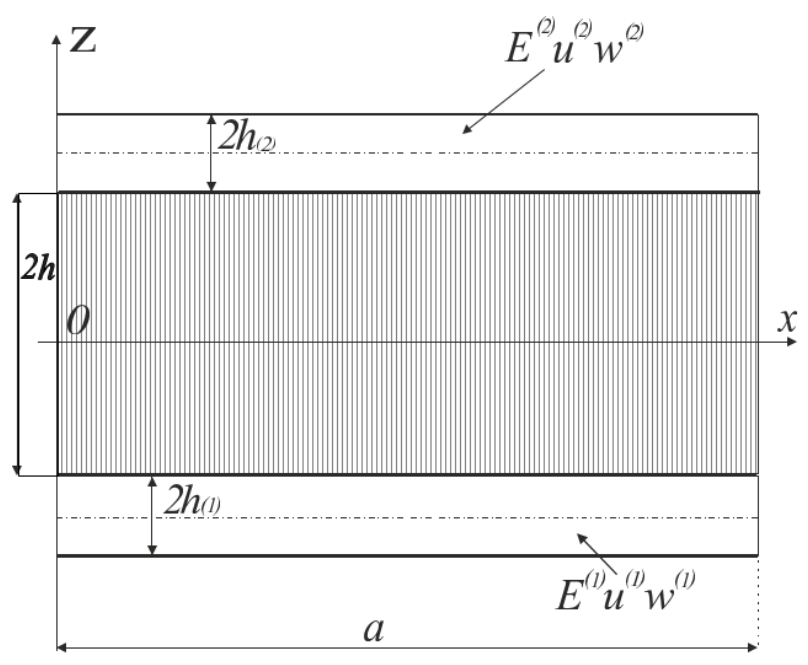

Рис 3. Поперечное сечение трехслойной пластины. 
Края пластины предполагаем закрепленными, так что выполняются условия $u^{(k)}(x)=0, w^{(k)}(x)=0, d w^{(k)} / d x=0$ при $x=0, x=a$.

Будем рассматривать задачу в геометрически нелинейной постановке, т.е.

$T_{(k)}^{11}=B_{(k)}\left(d u^{(k)} / d x+\frac{1}{2}\left(d w^{(k)} / d x\right)^{2}\right) \quad M_{(k)}^{11}=D_{(k)} d^{2} u^{(k)} / d x^{2}$

где

$B_{(k)}=2 h_{(k)} E^{(k)} /\left(1-v_{12}^{(k)}\right)\left(1-v_{21}^{(k)}\right)$ - жесткость k-го слоя на растяжение-сжатие, $E^{(k)}$ и $v_{12}^{(k)}, v_{21}^{(k)}$ - модуль упругости первого рода и коэффициенты Пуассона материала k-го несущего слоя, $D_{(k)}=B_{(k)} h_{(k)}^{2} / 3$ - изгибная жесткость k-го слоя. Пусть $U=\left(w^{(1)}, w^{(2)}, u^{(1)} w^{(2)}\right)$ - вектор перемещений точек срединной поверхности k-го слоя, $q^{1}$ - касательные напряжения в заполнителе. Для $q^{1}$ предполагаем выполненными граничные условия $q^{1}(0)=q^{1}(a)=0$.

В [12-14] для описания напряженно-деформированного состояния трехслойной пластины был построен следующий функционал потенциальной энергии деформаций: $L\left(U, q^{1}\right)=P\left(U, q^{1}\right)-A\left(U, q^{1}\right)-A_{q}\left(U, q^{1}\right)$, где

$$
\begin{aligned}
P\left(U, q^{1}\right)=\frac{1}{2} \int_{0}^{a}\{ & \sum_{k=1}^{2}\left[B_{(k)}\left(d u^{(k)} / d x+\frac{1}{2}\left(d w^{(k)} / d x\right)^{2}\right)^{2}+D_{(k)}\left(d^{2} w^{(k)} / d x^{2}\right)^{2}\right]+ \\
& \left.+c_{1}\left(q^{1}\right)^{2}+c_{2}\left(d q^{1} / d x\right)^{2}+c_{3}\left(w^{(2)}-w^{(1)}\right)^{2}\right\} d x
\end{aligned}
$$

есть потенциальная энергия деформации, $G_{13}, E_{3}$ - модули поперечного сдвига и обжатия заполнителя, $c_{1}=2 h / G_{13}, c_{2}=h^{3} / 3 E_{3}, c_{3}=E_{3} /(2 h)$,

$$
A\left(U, q^{1}\right)=\int_{0}^{a} \sum_{k=1}^{2}\left[X_{(k)}^{1} u^{(k)}+M_{(k)}^{1} d w^{(k)} / d x+X_{(k)}^{3} w^{(k)}\right] d x
$$

есть работа заданных внешних сил и моментов, $M_{(k)}^{1}-$ поверхностный момент внешних сил, приведенный к срединной поверхности k-го слоя, 


$$
A_{q}\left(U, q^{1}\right)=\int_{0}^{a}\left[\left(u^{(1)}-u^{(2)}\right)-\sum_{k=1}^{2} H_{(k)} d w^{(k)} / d x+c_{1} q^{1}-c_{2} d^{2} q^{1} / d x^{2}\right] q^{1} d x
$$

есть работа неизвестных контактных касательных напряжений на соответствующих перемещениях.

При этом установлено [15], что решение задачи о равновесии трехслойной пластины есть стационарные точки функционала L.

Предполагаем, что все постоянные ${ }^{B_{(k)}}, D_{(k)}, c_{1}, c_{2}, c_{3}, G_{13}, E_{3}, E^{(k)}$, $v_{12}^{(k)}, v_{21}^{(k)}, h, h_{(k)}$ положительны.

\section{2. Исследование корректности постановки задачи.}

Обозначим через $V_{k}=\stackrel{o(k)}{W_{2}}(0, a)$ пространства Соболева со скалярными произведениями $(u, \eta)_{k}=\int_{0}^{a} d^{k} u / d x^{k} d^{k} \eta / d x^{k} d x$, через $V_{q}$ - пространство Соболева функций, имеющих компактный носитель на $(0, a)$ и первую обобщенную производную, суммируемую с квадратом, со скалярным произведением

$$
(y, z)_{q}=\int_{0}^{a}\left[c_{1} y(x) z(x)+c_{2} d y / d x d z / d x\right] d x .
$$

Положим $V=V_{2} \times V_{2} \times V_{1} \times V_{1}$, Скалярное произведение в $\mathrm{V}$ будем обозначать через $(\cdot,)_{V}$. Докажем, что функционал $\mathrm{L}$ корректно определен на $V_{1} \times V_{q}$.

Перепишем (с учетом граничных условий для $q^{1}$ ) функционал L в виде

$$
\begin{gathered}
L\left(U, q^{1}\right)=\Phi_{0}(U)+\Phi_{1}\left(U, q^{1}\right)-\Phi_{2}\left(q^{1}\right), \text { где } \\
\Phi_{0}(U)=\frac{1}{2} \int_{0}^{a}\left\{\sum_{k=1}^{2}\left[B_{(k)}\left(d u^{(k)} / d x+\frac{1}{2}\left(d w^{(k)} / d x\right)^{2}\right)^{2}+D_{(k)}\left(d^{2} w^{(k)} / d x^{2}\right)^{2}\right]+\right. \\
\left.+c_{3}\left(w^{(2)}-w^{(1)}\right)^{2}\right\} d x-\int_{0}^{a} \sum_{k=1}^{2}\left[X_{(k)}^{1} u^{(k)}+M_{(k)}^{1} d w^{(k)} / d x+X_{(k)}^{3} w^{(k)}\right] d x, \\
\Phi_{1}\left(U, q^{1}\right)=\int_{0}^{a}\left[\sum_{k=1}^{2} H_{(k)} d w^{(k)} / d x+\left(u^{(2)}-u^{(1)}\right)\right] q^{1} d x,
\end{gathered}
$$




$$
\Phi_{2}\left(q^{1}\right)=\frac{1}{2} \int_{0}^{a}\left[c_{1}\left(q^{1}\right)^{2}+c_{2}\left(d q^{1} / d x\right)^{2}\right] d x=\frac{1}{2}\left\|q^{1}\right\|_{q}^{2} .
$$

При исследовании корректности постановки задачи нам потребуется следующая теорема вложения Соболева [16, стр.68].

Теорема 1. Пусть $\Omega \subset R^{n}$ - ограниченная область с регулярной границей $\Gamma$ и $1 \leq p<\infty$. Тогда $W_{p}^{(k)}(\Omega) \subset W_{r}^{(j)}(\Omega)$ для $0 \leq j<k$ и каждого $r$, удовлетворяющего условию $1 / p-(k-j) / n \leq 1 / r<1$; кроме того, для любой функции $u \in W_{p}^{(k)}(\Omega)$ справедливо неравенство

$$
\|u\|_{j, r} \leq C_{j r}^{k p}\|u\|_{k, p}
$$

где $\|\cdot\|_{k, p}$ - норма в $W_{p}^{(k)}(\Omega)$, а постоянная $C_{j r}^{k p}$ зависит от $\Omega, j, k, p, r$.

Докажем, что функционал $\Phi_{0}$ корректно определен на $V, \Phi_{1}$ корректно определен на $V \times V_{q}, \Phi_{2}$ корректно определен на $V_{q}$.

Лемма 1. Пусть $X_{(k)}^{1}, X_{(k)}^{3}, M_{(k)}^{1} \in L_{2}(0, a)$. Тогда для любой функции $U \in V$, справедливо неравенство

$$
\left|\Phi_{0}(U)\right| \leq \sum_{k=1}^{2}\left[d_{0}^{k}\left\|w^{(k)}\right\|_{2}+d_{1}^{k}\left\|w^{(k)}\right\|_{2}^{2}+d_{2}^{k}\left\|w^{(k)}\right\|_{2}^{4}+d_{3}^{k}\left\|u^{(k)}\right\|_{1}+d_{4}^{k}\left\|u^{(k)}\right\|_{1}^{2}\right](2)
$$

где $\quad d_{0}^{k}=\left\|M_{(k)}^{1}\right\|_{0} C_{12}^{22}+\left\|X_{(k)}^{3}\right\|_{0} C_{02}^{22}, \quad d_{1}^{k}=D_{(k)}+2 c_{3}\left(C_{02}^{22}\right)^{2}, \quad d_{2}^{k}=B_{(k)}\left(C_{12}^{22}\right)^{4}$, $d_{3}^{k}=\left\|X_{(k)}^{1}\right\|_{0} C_{02}^{21}, d_{4}^{k}=B_{(k)}$.

Доказательство. В дальнейшем норму в $L_{2}(0, a)$ будем обозначать через $\|\cdot\|_{0}$. Применяя неравенство $(\alpha+\beta)^{2} \leq 2\left(\alpha^{2}+\beta^{2}\right)$ и неравенство КошиБуняковского, имеем

$$
\begin{aligned}
& \left|\Phi_{0}(U)\right| \leq \frac{1}{2} \int_{0}^{a} \sum_{k=1}^{2}\left[B_{(k)}\left(2\left(\frac{d u^{(k)}}{d x}\right)^{2}+\frac{1}{2}\left(\frac{d w^{(k)}}{d x}\right)^{4}\right)+D_{(k)}\left(\frac{d^{2} w^{(k)}}{d x^{2}}\right)^{2}\right] d x+ \\
& +c_{3} \frac{1}{2} \int_{0}^{a}\left(\left(w^{(2)}\right)^{2}+\left(w^{(1)}\right)^{2}\right) d x+\sum_{k=1}^{2}\left[\int_{0}^{a}\left(X_{(k)}^{1}\right)^{2} d x\right]^{1 / 2}\left[\int_{0}^{a}\left(u^{(k)}\right)^{2} d x\right]^{1 / 2}+
\end{aligned}
$$




$$
\begin{gathered}
+\sum_{k=1}^{2}\left[\int_{0}^{a}\left(X_{(k)}^{3}\right)^{2} d x\right]^{1 / 2}\left[\int_{0}^{a}\left(w^{(k)}\right)^{2} d x\right]^{1 / 2}+\sum_{k=1}^{2}\left[\int_{0}^{a}\left(M_{(k)}^{1}\right)^{2} d x\right]^{1 / 2} \times \\
\times\left[\int_{0}^{a}\left(\frac{d^{2} w^{(k)}}{d x^{2}}\right)^{2} d x\right]^{1 / 2}=\sum_{k=1}^{2}\left[B_{(k)}\left\|u^{(k)}\right\|_{1,2}^{2}+\frac{1}{2} B_{(k)}\left\|w^{(k)}\right\|_{1,4}^{4}+\right. \\
+D_{(k)}\left\|w^{(k)}\right\|_{2,2}^{2}+2 c_{3}\left\|w^{(2)}\right\|_{0,2}^{2}+2 c_{3}\left\|w^{(1)}\right\|_{0,2}^{2}+ \\
+\sum_{k=1}^{2}\left\|X_{(k)}^{1}\right\|_{0}\left\|u^{(k)}\right\|_{0,2}+\sum_{k=1}^{2}\left\|X_{(k)}^{3}\right\|\left\|_{0}\right\| w^{(k)}\left\|_{0,2}+\sum_{k=1}^{2}\right\| M_{(k)}^{1}\|\|_{0}\left\|w^{(k)}\right\|_{1,2} .
\end{gathered}
$$

По определению $\left\|u^{(k)}\right\|_{1,2}=\left\|u^{(k)}\right\|_{1},\left\|w^{(k)}\right\|_{2,2}=\left\|w^{(k)}\right\|_{2}$. Далее, в силу (1) $\left\|w^{(k)}\right\|_{1,4} \leq C_{14}^{22}\left\|w^{(k)}\right\|_{2,2}=C_{14}^{22}\left\|w^{(k)}\right\|_{2},\left\|w^{(k)}\right\|_{0,2} \leq C_{02}^{22}\left\|w^{(k)}\right\|_{2,2}=C_{02}^{22}\left\|w^{(k)}\right\|_{2}$, $\left\|u^{(k)}\right\|_{0,2} \leq C_{02}^{12}\left\|u^{(k)}\right\|_{1,2}=C_{02}^{12}\left\|u^{(k)}\right\|_{1},\left\|w^{(k)}\right\|_{1,2} \leq C_{12}^{22}\left\|w^{(k)}\right\|_{2,2}=C_{12}^{22}\left\|w^{(k)}\right\|_{2}$.

С учетом этих оценок окончательно получаем требуемое неравенство (2).

Замечание 1. Результат леммы справедлив и для менее гладких функций $X_{(k)}^{1}, X_{(k)}^{3} w^{(k)}, M_{(k)}^{1}$.

Лемма 2. Для любых $U \in V, q^{1} \in V_{q}$ справедливо неравенство

$$
\left|\Phi_{1}(U)\right| \leq \sum_{k=1}^{2}\left[d_{5}\left\|u^{(k)}\right\|_{1}+d_{6}^{k}\left\|w^{(k)}\right\|_{2}\right]\left\|q^{1}\right\|_{q}
$$

где $d_{5}=c_{1}^{-1 / 2} C_{02}^{12}, d_{6}^{k}=c_{1}^{-1 / 2} C_{12}^{22} H_{(k)}$.

Доказательство. Имеем

$$
\begin{gathered}
\left|\Phi_{1}\left(U, q^{1}\right)\right| \leq \int_{0}^{a}\left[\sum_{k=1}^{2} H_{(k)}\left|d w^{(k)} / d x\right|+\left|u^{(2)}-u^{(1)}\right|\right]\left|q^{1}\right| d x \leq \\
\quad \leq \sum_{k=1}^{2}\left[H_{(k)}\left\|w^{(k)}\right\|_{1,2}\left\|q^{1}\right\|_{0}+\left(\left\|u^{(2)}\right\|_{0}+\left\|u^{(1)}\right\|_{0}\right)\left\|q^{1}\right\|_{0}\right]
\end{gathered}
$$

Далее, в силу теоремы $1\left\|u^{(k)}\right\|_{0} \leq C_{02}^{12}\left\|u^{(k)}\right\|_{1},\left\|w^{(k)}\right\|_{1,2} \leq C_{12}^{22}\left\|w^{(k)}\right\|_{2}$, кроме того,

$$
\left\|q^{1}\right\|_{0}^{2} \leq \frac{1}{c_{1}} \int_{0}^{a}\left[c_{1}\left|q^{1}\right|^{2}+c_{2}\left|d q^{1} / d x\right|^{2}\right] d x=\frac{1}{c_{1}}\left\|q^{1}\right\|_{q}^{2} \text {. С учетом этих }
$$
неравенств и получаем оценку (3). 
Теорема 2. Пусть $X_{(k)}^{1}, X_{(k)}^{3}, M_{(k)}^{1} \in L_{2}(0, a)$. Тогда функционал $\Phi_{0}$ корректно определен на $\mathrm{V}$, функционалы $\mathrm{L},{ }^{\Phi}{ }_{1}$ корректно определены на $V \times V_{q}$, $\Phi_{2}$ корректно определен на $V_{q}$.

Доказательство. Корректность определения функционалов $\Phi_{0}, \Phi_{1}$ на соответствующих пространствах следует из лемм $1,2, \Phi_{2}$ корректно определен, ибо равен полуквадрату нормы, таким образом корректно определен на $V \times V_{q}$ и функционалы L.

\section{3. Заключение.}

В настоящей работе проведено исследование корректности обобщенной постановки геометрически нелинейной задачи об изгибе трехслойной пластины с трансверсально-мягким заполнителем. Решение задачи о равновесии трехслойной пластины есть стационарные точки корректно определенного функционала. Это позволит в дальнейшем сформулировать обобщенную постановку в виде операторного уравнения, исследовать ее разрешимость и разработать и исследовать приближенные методы решения полученного операторного уравнения. При этом предполагается использовать подходы, разработанные в [17-26] подходы.

Публикация осуществлена при финансовой поддержке РФФИ и Правительства Республики Татарстан в рамках научных проектов № 15-4102569, 15-01-05686, 16-38-00788. 


\section{Литература:}

1. Иванов В.А., Паймушин В.Н., Полякова Т.В. Уточненная теория устойчивости трехслойных конструкций (линеаризованные уравнения нейтрального равновесия и простейшие одномерные задачи) // Известия высших учебных заведений. Математика. - 1995. - № 3. - С. 15-24.

2. Иванов В.А., Паймушин В.Н. Уточнение уравнений динамики многослойных оболочек с трансверсально-мягким заполнителем // Известия Российской академии наук. Механика тв. тела. - 1995. - № 3. - С. $142-152$.

3. Паймушин В.Н., Шалашилин В.И. О геометрически нелинейных уравнениях теории безмоментных оболочек с приложениями к задачам о неклассических формах потери устойчивости цилиндра // Прикладная математика и механика. - 2006. - Т. 70, № 1. - С. 100-110.

4. Paimushin V.N., Polyakova N.V. The consistent equations of the theory of plane curvilinear rods for finite displacements and linearized problems of stability // Journal of Applied Mathematics and Mechanics. - 2009. - V. 73, № 2. - C. 220236.

5. Paimushin V.N., Firsov V.A., Gyunal I., Egorov A.G. Theoretical-experimental method for determining the parameters of damping based on the study of damped flexural vibrations of test specimens. 1. Experimental basis // Mechanics of Composite Materials. - 2014. - V. 50, № 2. - P. 127-136.

6. Бадриев И.Б., Макаров М.В., Паймушин В.Н. О взаимодействии композитной пластины, имеющей вибропоглощающее покрытие, с падающей звуковой волной // Известия высших учебных заведений. Математика. - 2015. - № 3. - С. 75-82.

7. Бадриев И.Б., Гарипова Г.З., Макаров М.В., Паймушин В.Н., Хабибуллин Р.Ф. О решении физически нелинейных задач о равновесии трехслойных пластин с трансверсально-мягким заполнителем // Ученые записки 
Казанского университета. Серия: Физико-математические науки. - 2015. Т. 157, № 1. - C. 15-24.

8. Бадриев И.Б., Бандеров В.В., Гарипова Г.З., Макаров М.В. О разрешимости нелинейной задачи о равновесии трехслойной пластины // Вестник Тамбовского университета. Серия: Естественные и технические науки. 2015. - T. 20, № 5. - C. 1034-1036.

9. Badriev I.B., Garipova G.Z., Makarov M.V., Paimushin V.N., Khabibullin R.F. Solving Physically Nonlinear Equilibrium Problems for Sandwich Plates with a Transversally Soft Core // Lobachevskii Journal of Mathematics. - 2015. - Vol. 36, № 4. - P. 474-481.

10. Badriev I.B., Banderov V.V., Garipova G.Z., Makarov M.V., Shagidullin R.R. On the solvability of geometrically nonlinear problem of sandwich plate theory // Applied Mathematical Sciences. - 2015. - V. 9, № 82. - P. 4095-4102.

11. Бадриев И.Б., Бандеров В.В., Гарипова Г.З., Макаров М.В. Исследование задачи об определении НДС физически нелинейной задачи об изгибе трехслойной пластины // XI Всероссийский съезд по фундаментальным проблемам теоретической и прикладной механики (Казань, 20-24 августа 2015 г.): сборник трудов. - Казань: Издательство Казанского (Приволжского) федерального университета, 2015. - С. 276.

12. Паймушин В.Н., Бобров С.Н. Уточненная геометрически нелинейная теория трехслойных оболочек с трансверсально-мягким заполнителем средней толщины для исследования смешанных форм потери устойчивости // Механика композитных материалов. - 2000. - Т. 36, № 1. - С. 95-108.

13. Паймушин В.Н. К вариационным методам решения нелинейных пространственных задач сопряжения деформируемых тел // Доклады Академии наук. - 1983. - Т. 273, № 5. - С. 1083-1086.

14. Паймушин В.Н. Нелинейная теория среднего изгиба трехслойных оболочек с дефектами в виде участков непроклея // Прикладная механика. - 1987. T. 23, № 11. - C. 32-38. 
15. Паймушин В.Н. Обобщенный вариационный принцип Рейсснера в нелинейной механике пространственных составных тел с приложениями к теории многослойных оболочек // Известия Академии наук СССР. Механика твердого тела. - 1987. - № 2. - С. 171-180.

16. Adams R.A. Sobolev Spaces. - New York, San Francisco, London: Academic Press, 1975. - 286 p.

17. Бадриев И.Б., Карчевский М.М. Применение метода двойственности к решению нелинейных задач теории фильтрации с предельным градиентом // Дифференциальные уравнения. - 1982. - Т. 18, № 7. - С. 1133-1144.

18. Badriev I.B. On the solving of variational inequalities of stationary problems of two-phase flow in porous media // Applied Mechanics and Materials. - 2013. - V. 392. - P. 183-187.

19. Badriev I.B., Banderov V.V. Iterative methods for solving variational inequalities of the theory of soft shells // Lobachevskii Journal of Mathematics. 2014. - V. 35, № 4. - P. 371-383.

20. Badriev I.B., Banderov V.V. Numerical method for solving variation problems in mathematical physics // Applied Mechanics and Materials. - 2014. - V. 668-669. - P. 1094-1097.

21. Badriev, I.B., Shagidullin, R.R. A study of the convergence of a recursive process for solving a stationary problem of the theory of soft shells // Journal of Mathematical Sciences. - 1995. - V. 73, № 5. - P. 519-525.

22. Badriev, I.B., Karchevskii, M.M. Convergence of an iterative process in a Banach space // Journal of Mathematical Sciences. - 1994. - V. 71, № 6. - P. 2727-2735.

23. Бадриев И.Б., Нечаева Л.А. Математическое моделирование установившейся фильтрации с многозначным законом // Вестник Пермского национального исследовательского политехнического университета. Механика. - 2013. - № 3. - С. 35-62.

24. Бадриев И.Б., Желтухин В.С., Макаров М.В., Паймушин В.Н. Численное 
решение задачи о равновесии трехслойной пластины с трансверсальномягким заполнителем в геометрически нелинейной постановке // Вестник Казанского технологического университета. - 2014. - Т.17, № 23. - С. 393 396.

25. Бадриев И.Б., Бандеров В.В. Итерационные методы решения вариационных неравенств теории мягких оболочек // Ученые записки Казанского университета. Серия: Физико-математические науки. - 2013. - Т. 155, кн.2. - C. 18-32.

26. Badriev I.B., Banderov V.V., Makarov M.V., Paimushin V.N. Determination of stress-strain state of geometrically nonlinear sandwich plate // Applied Mathematical Sciences. - 2015. - V. 9, № 78. - P. 3887-3895. 